\title{
Characterization and Uncertainty Assessment of a Certified Reference Material of Chloramphenicol in Methanol (GBW(E)082557)
}

\author{
Mengrui Yang, Min Wang, Jian Zhou, Yinqing Song, and Tongtong Wang \\ Institute of Quality Standard and Testing Technology for Agro-Products, Chinese Academy of Agricultural Sciences, \\ Beijing 100081, China
}

Correspondence should be addressed to Min Wang; wangmincaas@126.com

Received 7 April 2016; Revised 26 May 2016; Accepted 14 June 2016

Academic Editor: Günther K. Bonn

Copyright (c) 2016 Mengrui Yang et al. This is an open access article distributed under the Creative Commons Attribution License, which permits unrestricted use, distribution, and reproduction in any medium, provided the original work is properly cited.

Prior to preparation of CRM candidate of chloramphenicol in methanol with a concentration of $100 \mathrm{mg} / \mathrm{L}$, two independent methods including mass balance $(\mathrm{MB})$ and quantitative nuclear magnetic resonance (qNMR) were employed to precisely measure the mass fraction of pure chloramphenicol materials. The mass fraction was assigned to be $99.8 \%$ with uncertainty of $0.3 \%$. Homogeneity testing and stability study of chloramphenicol in methanol were examined by using high performance liquid chromatography. Additionally, the uncertainties originating from the process of CRM development were comprehensively evaluated. The experimental results indicate that the property value of this $\mathrm{CRM}$ is homogeneous and stable at $4^{\circ} \mathrm{C}$ for at least six months. The new CRM (GBW(E)082557) can be applicable to calibration of instrument and assurance of accuracy and comparability of results in routine measurement.

\section{Introduction}

A certified reference material (CRM), which is known as one kind of substance or material possessing one or more homogenous and stable property values with uncertainties under a specific confidence level, plays a very important role in the metrological sciences referring to instrument calibration, analytical method validation, and quantity transfer and traceability [1]. Therefore, precise and accurate assignment of property value should be a priority of all in the development of a CRM. To the pure organic substance, a series of measuring approaches are recognized and recommended by Bureau International des Poids et Mesures (BIPM). Mass balance (MB) method as an indirect purity measuring means is implemented by combination of several conventional instrumental measurements including chromatographic analysis of organic compounds, Karl-Fischer titration for water, inductively coupled plasma-mass spectrometry and atom absorbance spectroscopy for inorganic ions, and Thermal
Gravity Analysis (TGA) for nonvolatile inorganic impurities [2-4]. In addition, differential scanning calorimetry (DSC) on the basis of the melting point depression caused by impurities is usually applicable to accurate purity measurement, while the impurities are soluble in the melting status and the true purity of main component is more than 98.5\% [5-7]. Recently, quantitative nuclear magnetic resonance (qNMR) as a rising technique was proposed by BIPM for purity assay, which can be directly determined by using ideal internal standard. The qNMR approach is frequently applied in the metrology field and can be regarded as a primary method [8]. Therefore, it becomes more and more popular in purity measurement of organic compounds [9-12]. However, the selection of an internal standard is important and critical in the purity measurement. First of all, the selected internal standard must be a CRM to assure the traceability of measurement result to SI unit. Secondly, it must be soluble in common solvent and have no interference peaks with the target substance. Additionally, it is very important that 
the pointed signal peak of main component should be suitable and have no interference coming from the structurally related organic impurities and the internal standard.

Chloramphenicol, which was a common and famous antibiotic drug, has already been forbidden in the breeding process of livestock and poultry, because the drug residue in agroproducts could lead to toxic side effect to human body through food chain. Therefore, a series of testing standards for determination of chloramphenicol residue in honey, animal products, and animal feed were issued. Meanwhile, chloramphenicol CRMs have been shown to be important and essential in the implementation of these testing standards. Although various commercial CRMs associated with chloramphenicol are available according to the information from COMAR database (international database for certified reference materials), such as purity CRM of chloramphenicol from NIM (National Institute of Metrology, China) and matrix CRMs of chloramphenicol in pork and in honey from ERMM, diversity of chloramphenicol CRMs not only are enrichment for CRM database but also provide more selectable and suitable CRMs for routine work in testing laboratories. Prior to CRM development, a survey of utilization of chloramphenicol CRM in the testing standards was carried out. It was found that the calibration solution was usually prepared individually in each test laboratory by dissolving pure chloramphenicol CRM into methanol or acetonitrile with a concentration of $\sim 100 \mathrm{mg} / \mathrm{L}$. To reduce the operational steps and minimize the error from preparation of calibration solution in each laboratory, a user-friendly and timesaving CRM of chloramphenicol in methanol would be welcomed.

In this paper, the mass fraction of chloramphenicol material was precisely determined by using qNMR approach. To confirm the measurement result, $\mathrm{MB}$ as another independent method was carried out on purity determination. As mass fraction of chloramphenicol was accurately assigned, CRM of chloramphenicol in methanol was first developed strictly according to ISO Guide 34 and 35 and JJF 1006-1994 (Chinese Technical Norm of Primary Reference Material) [13-15]. Additionally, details on establishment of $\mathrm{qNMR}$ and $\mathrm{MB}$ methods for mass fraction assignment of chloramphenicol, preparation of chloramphenicol in methanol, homogeneity test and stability study, and uncertainty evaluation were sufficiently described.

\section{Materials and Methods}

2.1. Materials. All the high purity organic solvents were purchased from Merk. Certified reference materials of ethyl paraben (GBW(E) 100064) with a certificate value of $99.7 \%$ as an internal standard for qNMR measurement and chloramphenicol (GBW(E)060907) with a certificate value of $99.8 \%$ were supplied by National Institute of Metrology (NIM), China. Methanol- $\mathrm{D}_{4}$ was obtained from Sigma-Aldrich. The powder of chloramphenicol material with a labeled purity of 99.8\% was purchased from Tokyo Chemical Industry (TCI) Co., Ltd., Japan.
2.2. Apparatus. A high performance liquid chromatography system (Shimadzu, Japan) equipped with Agilent Zorbax XDB-C18 $(250 \mathrm{~mm} \times 4.6 \mathrm{~mm}, 5.0 \mu \mathrm{m})$ column and UV detector was applied in homogeneity test, stability study, and measurement of main component and unknown organic impurities. The Karl Fischer titration (METTLER TOLEDO DL39) was used for measurement of water content in chloramphenicol raw material. The analysis of nonvolatile impurities and volatile organic impurities was carried out on Thermal Gravity Analysis (PerkinElmer Pyris1, US) and gas chromatography coupled with flame ionization detector (GCFID), respectively. A Bruker Avance III spectrometer with a cryoprobe at $400 \mathrm{MHz}$ was employed to measure the mass fraction of chloramphenicol. All samples were weighed by using a Mettler AL104 analytical balance with a metrological verification certificate. Qualitative analysis was performed on a Bruker VERTEX 70 Infrared spectroscopy and Agilent mass spectrometry, respectively.

\subsection{Purity Determination of Chloramphenicol}

2.3.1. Mass Balance Methods. The purity determination using mass balance method can be described with the following equation:

$$
P_{\mathrm{MB}}=P_{0}\left(100 \%-X_{\mathrm{w}}-X_{\mathrm{n}}-X_{\mathrm{v}}\right) \times 100 \%,
$$

where $P_{0}$ is main content determined by using HPLC area normalization method and $X_{\mathrm{w}}$ is the mass fraction of water; $X_{\mathrm{v}}$ is the mass fraction of volatile impurities; and $X_{\mathrm{n}}$ is the mass fraction of nonvolatile inorganic impurities.

The main contents and organic impurities of chloramphenicol were determined by using HPLC area normalization method, in which an Agilent Zorbax XDB-C18 $(250 \times$ $4.6 \mathrm{~mm}, 5 \mu \mathrm{m}$ ) column was used and UV detector was set at $280 \mathrm{~nm}$ wavelength. The mobile phase was consisted of methanol and water adjusted with $0.2 \%$ acetic acid at a ratio of $40: 60$. The injection volume and the column oven temperature were $10 \mu \mathrm{L}$ and $30^{\circ} \mathrm{C}$, respectively. In addition, the mass fraction of water $\left(X_{\mathrm{w}}\right)$, volatile impurities $\left(X_{\mathrm{v}}\right)$, and nonvolatile inorganic impurities $\left(X_{n}\right)$ were measured using a Karl Fischer titration, the headspace gas chromatography combined with FID detector, and the Thermal Gravity Analysis, respectively.

2.3.2. Quantitative Nuclear Magnetic Resonance. The purity of chloramphenicol $\left(P_{\mathrm{NMR}}\right)$ measured by qNMR can be calculated by the following equation:

$$
P_{\mathrm{NMR}}=\frac{I_{x}}{I_{\mathrm{std}}} \cdot \frac{n_{\mathrm{std}}}{n_{x}} \cdot \frac{M_{x}}{M_{\mathrm{std}}} \cdot \frac{m_{\mathrm{std}}}{m_{x}} \cdot P_{\mathrm{std}},
$$

where $I_{x}$ and $I_{\text {std }}$ are integrated peak area of chloramphenicol and internal standard, respectively; $n_{\text {std }}$ and $n_{x}$ are spin numbers of internal standard and chloramphenicol, respectively; $M_{x}$ and $M_{\text {std }}$ are molecular weights of chloramphenicol and internal standard, respectively; $m_{\text {std }}$ and $m_{x}$ are mass of internal standard and chloramphenicol in a determined sample. $P_{\text {std }}$ is purity of internal standard. 


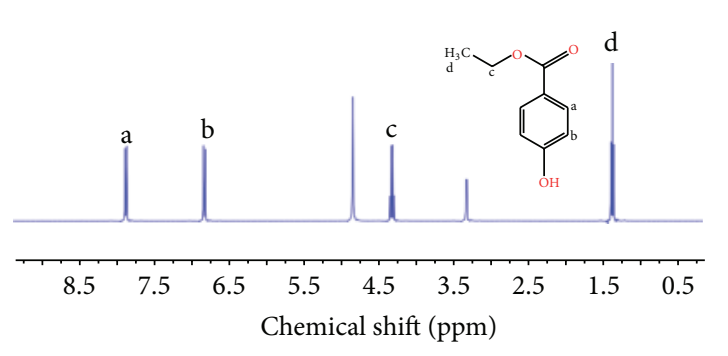

(a) Ethyl paraben

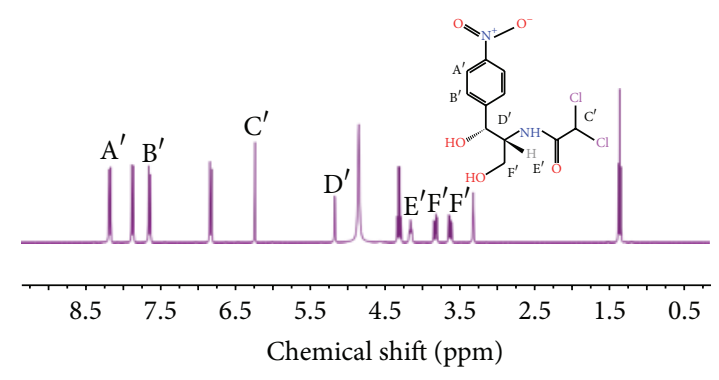

(b) Ethyl paraben + chloramphenicol

FIGURE 1: The H-NMR spectra of (a) internal standard (ethyl paraben and methanol- $\mathrm{D}_{4}$ ) and (b) sample solution (chloramphenicol, ethyl paraben, and methanol- $\mathrm{D}_{4}$ ).

The purity measurement was performed on Bruker Avance III $400 \mathrm{MHz}{ }^{1} \mathrm{H}$ qNMR spectrometer which was equipped with pulsed field gradient probe at temperature of $293.4 \mathrm{~K}$. The setting parameters are as follows: the pulse width of $3.98 \mathrm{~s}$ at $90^{\circ}$ spectral band width of $8223.43 \mathrm{~Hz}$, delay time of $60 \mathrm{~s}$, and the number of transitions of 16. A sample including $10 \mathrm{mg}$ ethyl paraben CRM as internal standard and $20 \mathrm{mg}$ chloramphenicol was dissolved in $0.75 \mathrm{~mL}$ methanol$\mathrm{D}_{4}$ solvent and was analyzed.

Acquisition on Bruker instrument was based on the command GO. During the NMR acquisition period, a steadystate pulse was applied prior to the relaxation decay. Sixteen dummy scans were acquired before data corrections. The receiver gain was automatically set by instrument. Each sample took about $10 \mathrm{~min}$ to finish. Seven samples were measured under the same conditions. All qNMR spectra were analyzed by using MestReNova software. A decay signal was automatically processed by Fourier transformation. The chemical shift of all spectra was referenced to residual ${ }^{1} \mathrm{H}$ signals in deuterated solvent at $3.31 \mathrm{ppm}$ for $\mathrm{CD}_{3} \mathrm{OD}$. Phase correction and baseline correction were automatically applied according to the procedure of software guide. Peak integrations were set to an identical range for each spectrum.

2.4. Preparation of the CRM. According to the determined purity, the CRM of chloramphenicol in methanol was prepared by dissolving precisely weighted $100 \mathrm{mg}$ chloramphenicol into $1000 \mathrm{~mL}$ methanol under strictly controlled temperature and moisture. After sufficient mixing and dissolution, the CRM of chloramphenicol in methanol was dispensed into ampoule bottles with $1.0 \mathrm{~mL}$ per each. A batch of CRM candidates including about 1000 units was produced and was stored at $4^{\circ} \mathrm{C}$ condition.

2.5. Homogeneity and Stability Test. Homogeneity and stability test was carried out by using HPLC-UV approach. 25 bottles were randomly selected from the batch and were examined to test the between-bottle homogeneity of chloramphenicol in methanol. Each bottle was measured in triplicate for testing within-bottle homogeneity. The homogeneity testing results were statistically analyzed by one-way analysis of variance (ANOVA).
The short-term and long-term stability testing was evaluated in eight days and six months, respectively. For short-term stability, the CRM bottles were exposed at $20^{\circ} \mathrm{C}$ and $40^{\circ} \mathrm{C}$ on predetermined days of $2,4,6$, and 8 ; for long-term stability, the CRM bottles stored at $4^{\circ} \mathrm{C}$ conditions were analyzed in the 1st, 3rd, and 6th months. A new chloramphenicol calibration solution was freshly prepared when the stability was tested. At each time, a calibration solution and two bottles of CRM were analyzed by HPLC-UV method. The data of stability testing was assessed by regression analysis.

\section{Results and Discussion}

3.1. Purity Determination. Quantitative nuclear magnetic resonance (qNMR) as a characterization approach for purity measurement was recently recommended by BIPM. To obtain precise and accurate results of purity, several principle rules should be noticed in the measurement processes. Measurement conditions including phase correction, baseline correction, pulse intervals, and spectral window were systematically optimized. First, a certified reference material as an internal standard is necessary to realize the traceability from testing result to SI unit. Second, the selected internal standard and target analyte should be simultaneously soluble in the same and common deuterium labeled solvent. Third, the pointed signal peaks for quantification must have no interference for each other. Ethyl paraben CRM was commonly thought of as an ideal internal standard for purity measurement in many researches; thus, the ethyl paraben was selected in this study. In comparison with spectrum of only ethyl paraben in Figure 1(a), $\mathrm{H}$ peaks ascribed to internal standard and analyte can be easily identified in Figure 1(b) which shows the H-NMR spectrum of mixture of ethyl paraben and chloramphenicol in methanol- $\mathrm{D}_{4}$. As shown in Table 1, three pairs of peaks at different chemical shifts with no overlap were chosen for quantitative analysis of chloramphenicol; however, different purity results ranging from $98.94 \%$ to $99.82 \%$ were obtained according to the equation in Section 2.3.2. To judge which result is more accurate and reliable, the baseline of NMR spectrum was zoomed up as shown in Figure 2. It is found that only the two peaks in pair A are in the same level of baseline as shown in cycle 1 and cycle 2 . However, there are additional 
TABLE 1: Mass fraction of chloramphenicol calculated with different pointed signal peaks $(n=7)$.

\begin{tabular}{lcccc}
\hline Pairs & Peak of $I_{x} / \mathrm{ppm}$ & Peak of $I_{\text {std }} / \mathrm{ppm}$ & Mean of $P_{\mathrm{NMR}} / \%$ & SD $/ \%$ \\
\hline A & $\mathrm{d}$ & $\mathrm{F}^{\prime}$ & 99.82 & 0.07 \\
B & $\mathrm{c}$ & $\mathrm{F}^{\prime}$ & 99.13 & 0.09 \\
C & $\mathrm{a}$ & $\mathrm{A}^{\prime}$ & 98.94 & 0.05 \\
\hline
\end{tabular}

" $n$ " means different data sets measured under the same conditions.

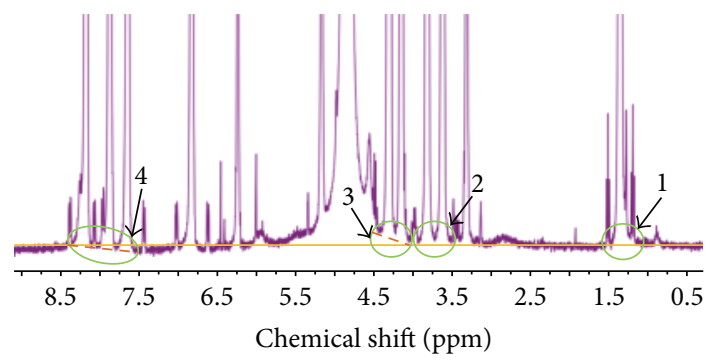

FIGURE 2: Baseline enlarged H-NMR spectrum of sample solution (chloramphenicol, ethyl paraben, and methanol- $\mathrm{D}_{4}$ ).

tiny peaks in cycles 1,3 , and 4 , which are considered to be from the structurally related organic impurity by comparing with spectrum of internal standard only. Due to the inevitable interference from impurities, a commercial chloramphenicol CRM was used for checking the impurities and evaluating its influence on value assignment. Although the similar structurally related organic impurity was also observed, its contribution to peak integration in qNMR can be ignored. In this case, it was presumed that selection of quantitative peaks on the basis of same baseline level might be more critical in this study. Therefore, the purity of $99.82 \%$ was considered to be the closest to the true value among the three results.

For further confirmation, mass balance (MB) method was carried out on the measurement of purity. On the contrary with $\mathrm{qNMR}, \mathrm{MB}$ is an indirect purity measurement method and is accomplished by precise measurements of impurities including organic impurities, moisture, and volatile and nonvolatile impurities. The analysis of organic impurities is generally measured by using chromatography. In this research, HPLC-UV was performed to analyze organic impurities in chloramphenicol. In order to identify and measure impurities sufficiently and comprehensively, methanol (blank) and chloramphenicol in methanol with concentrations of $100 \mathrm{mg} / \mathrm{L}$ and $2000 \mathrm{mg} / \mathrm{L}$ were determined on the UV absorbance at $280 \mathrm{~nm}$. In comparison with methanol blank, peaks of chloramphenicol and impurities were observed and separated in Figure 3. A total of seven impurities peaks were detected at the concentration of $2000 \mathrm{mg} / \mathrm{L}$, while two peaks (impurity 4 and impurity 7) were obviously observed at a concentration of $100 \mathrm{mg} / \mathrm{L}$ as shown in Figures 3(b) and 3(c). It should be emphasized that the concentration for the purity measurement by using HPLC area normalization method must be prepared as high as possible to guarantee signal response of all impurities sufficiently. Therefore, the purity determined by HPLC-UV was $99.84 \%$ with a standard deviation of $0.02 \%$ shown in Table 2. For the water measurement, a Karl-Fischer

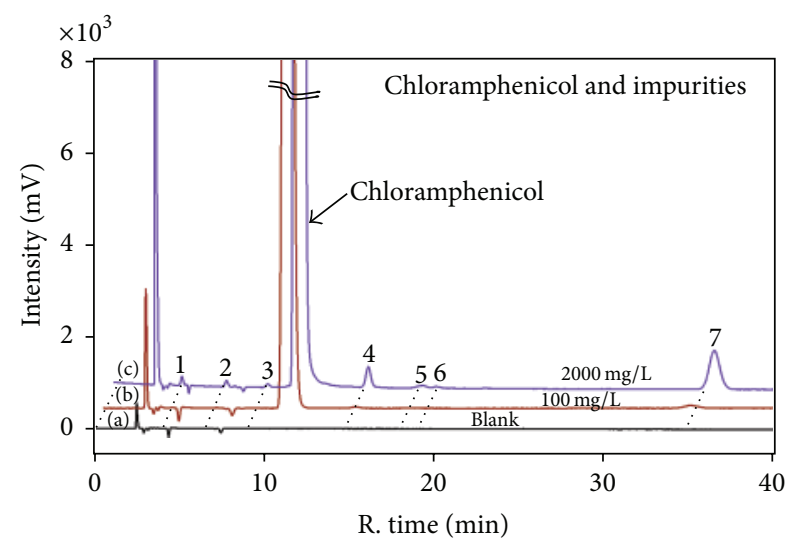

Figure 3: Chromatography of (a) methanol blank, (b) $100 \mathrm{mg} / \mathrm{L}$ chloramphenicol in methanol, and (c) $2000 \mathrm{mg} / \mathrm{L}$ chloramphenicol in methanol.

coulometric titrator was used. Mass fraction of water was determined and was calculated to be $0.025 \%$ with standard deviation of $0.003 \%$ as shown in Table 2 . In this study, the nonvolatile inorganic impurities were measured by heating the samples from $35^{\circ} \mathrm{C}$ to $600^{\circ} \mathrm{C}$ at $10^{\circ} \mathrm{C} / \mathrm{min}$. Gradual evaporation process curve was obtained and the weight of sample did not change by heating up to $600^{\circ} \mathrm{C}$. The volatile organic impurities were determined by GC-FID. The headspace gas chromatography combined with FID detector was carried out on a column of DB-624 $(30 \mathrm{~m} \times 0.32 \mathrm{~mm}, 1.8 \mu \mathrm{m})$ to determine volatile impurities associated with twelve kinds of conventional solvents including methanol and acetonitrile. Parameters were set as follows: hydrogen, $40 \mathrm{~mL} / \mathrm{min}$, air, $400 \mathrm{~mL} / \mathrm{min}$, transfer line temperature, $100^{\circ} \mathrm{C}$, and injection volume, $50 \mu \mathrm{L}$. The initial temperature was $45^{\circ} \mathrm{C}$ and it was held for $5 \mathrm{~min}$. The temperature was increased to $120^{\circ} \mathrm{C}$ at $7^{\circ} \mathrm{C} / \mathrm{min}$ and to $230^{\circ} \mathrm{C}$ at $15^{\circ} \mathrm{C} / \mathrm{min}$ and was held for $8 \mathrm{~min}$. It was found that several peaks with intensity less than 3 times signal-to-noise ratio (LOD) were detected. Therefore, the mass fraction of nonvolatile inorganic and volatile organic impurities can be ignored.

3.2. Homogeneity Test. For the homogeneity test, 25 ampoule bottles of samples were chosen from the batch of CRMs randomly and were determined in triplicates for each by using optimized HPLC-UV method mentioned in the above section. The area of signal peaks was recorded by HPLC-UV area normalization method as shown in Table 3. According to General and Statistical Principles for Characterization of Reference Material JJF 1343-20012, the data was analyzed by one-way analysis of variance (ANOVA) and the results were 
TABLE 2: Mass fraction of chloramphenicol measured by $\mathrm{MB}$ methods.

\begin{tabular}{lccc}
\hline Sample vials number & HPLC/\% & Moisture/\% & Purity/\% \\
\hline 1 & 99.87 & 0.020 & 99.85 \\
2 & 99.84 & 0.030 & 99.81 \\
3 & 99.84 & 0.026 & 99.81 \\
4 & 99.87 & 0.025 & 99.85 \\
5 & 99.83 & 0.028 & 99.80 \\
6 & 99.83 & 0.023 & 99.81 \\
7 & 99.82 & 0.024 & 99.80 \\
Mean & 99.84 & 0.025 & 99.82 \\
SD & 0.020 & 0.003 & 0.02 \\
\hline
\end{tabular}

TABle 3: Data of homogeneity study.

\begin{tabular}{|c|c|c|c|c|c|}
\hline \multirow{2}{*}{ Vials number } & \multicolumn{5}{|c|}{ Peak area } \\
\hline & 1 & 2 & 3 & Mean & SD \\
\hline 1 & 3377539 & 3455815 & 3426396 & 3419917 & 39538 \\
\hline 2 & 3363161 & 3383494 & 3413952 & 3386869 & 25563 \\
\hline 3 & 3373464 & 3438840 & 3444717 & 3419007 & 39551 \\
\hline 4 & 3361335 & 3400715 & 3431809 & 3397953 & 35318 \\
\hline 5 & 3361386 & 3385011 & 3400994 & 3382464 & 19926 \\
\hline 6 & 3370750 & 3440166 & 3441286 & 3417401 & 40405 \\
\hline 7 & 3396404 & 3442398 & 3445094 & 3427965 & 27366 \\
\hline 8 & 3412038 & 3456559 & 3452226 & 3440274 & 24549 \\
\hline 9 & 3492616 & 3414137 & 3400516 & 3435756 & 49711 \\
\hline 10 & 3412637 & 3423430 & 3431176 & 3422414 & 9311 \\
\hline 11 & 3382569 & 3410793 & 3415548 & 3402970 & 17827 \\
\hline 12 & 3351945 & 3362416 & 3401855 & 3372072 & 26319 \\
\hline 13 & 3359186 & 3400385 & 3432492 & 3397354 & 36747 \\
\hline 14 & 3363789 & 3419854 & 3419894 & 3401179 & 32381 \\
\hline 15 & 3373310 & 3417544 & 3432514 & 3407789 & 30784 \\
\hline 16 & 3377488 & 3418379 & 3415865 & 3403911 & 22917 \\
\hline 17 & 3382255 & 3412441 & 3413938 & 3402878 & 17876 \\
\hline 18 & 3393284 & 3425055 & 3415432 & 3411257 & 16292 \\
\hline 19 & 3406204 & 3444518 & 3444612 & 3431778 & 22148 \\
\hline 20 & 3403316 & 3406961 & 3405709 & 3405329 & 1852 \\
\hline 21 & 3364827 & 3408684 & 3409946 & 3394486 & 25693 \\
\hline 22 & 3382216 & 3383257 & 3405014 & 3390162 & 12872 \\
\hline 23 & 3400230 & 3387406 & 3414257 & 3400631 & 13430 \\
\hline 24 & 3361051 & 3391923 & 3404463 & 3385812 & 22342 \\
\hline 25 & 3353175 & 3403030 & 3394614 & 3383606 & 26688 \\
\hline
\end{tabular}

shown in Table 4. It was found that the calculated $F$ value was less than the critical value of $F_{0.05}(24,50)$. It has been demonstrated that the homogeneity of chloramphenicol in methanol was homogenous under the minimum sampling of $10 \mu \mathrm{L}$.

\subsection{Stability Study}

3.3.1. Short-Term Stability Study. To assess the effect of temperature change in delivery, portions of samples were
TABLE 4: Results of homogeneity study.

\begin{tabular}{lc}
\hline Items & Results \\
\hline Total mean & 3405649 \\
Total SD & 28716 \\
Between-bottle & 946997904 \\
Within-bottle & 765842476 \\
$F$ & $F=s_{1}^{2} / s_{2}^{2}=1.24$ \\
$F$ critical value & $F_{0.05}(24,50)=1.740$ \\
Evaluation result & $F<F_{0.05}(24,50)$ \\
\hline
\end{tabular}

TABLE 5: Result of short-term stability study.

\begin{tabular}{lcc}
\hline \multirow{2}{*}{ Time/day } & \multicolumn{2}{c}{ Value $/(\mathrm{mg} / \mathrm{L})$} \\
\hline 0 & 100.7 & Condition $/ 40^{\circ} \mathrm{C}$ \\
2 & 100.2 & 100.7 \\
4 & 100.0 & 99.8 \\
6 & 99.4 & 96.4 \\
8 & 99.9 & 93.3 \\
\hline Mean & 99.8 & 92.4 \\
\hline$b_{1}$ & -0.119 & 96.6 \\
$b_{0}$ & 100.5 & -1.153 \\
$s^{2}$ & 0.1053 & 101.1 \\
$s\left(b_{1}\right)$ & 0.0513 & 0.7423 \\
$t_{0.95, n-2}$ & 3.18 & 0.1362 \\
Conclusion & $\left|b_{1}\right|<t_{0.95, n-2} \cdot s\left(b_{1}\right)$, & 3.18 \\
& stable & $\left|b_{1}\right|>t_{0.95, n-2} \cdot s\left(b_{1}\right)$, \\
\hline
\end{tabular}

prestored at $20^{\circ} \mathrm{C}$ and $40^{\circ} \mathrm{C}$ to simulate the shipment conditions. Two ampoule bottles of sample were stored at each temperature for 2, 4, 6, and 8 days. After the indicated storage periods, the samples and a freshly prepared chloramphenicol in methanol as calibration solution were determined in triplicate using the HPLC-UV method. The stability data at $20^{\circ} \mathrm{C}$ and $40^{\circ} \mathrm{C}$ were plotted against time, respectively, and the regression lines were calculated (shown in Table 5). The observed slope $b_{1}$ was tested for significance using $t$-test, with $s\left(b_{1}\right)$ being the uncertainty of slope and $t_{0.95, n-2}$ of critical $t$ value for a confidence level of $95 \%$ and $n-2$ degree of freedom. As shown in Table 3, no significance slope was found for storage temperatures of $20^{\circ} \mathrm{C}\left(\left|b_{1}\right|<t_{0.95, n-2}\right.$. $\left.s\left(b_{1}\right)\right)$, whereas the slope was highly significant for a storage temperature of $40^{\circ} \mathrm{C}\left(\left|b_{1}\right|>t_{0.95, n-2} \cdot s\left(b_{1}\right)\right)$. This led to a conclusion that if the shipment temperature was not higher than $20^{\circ} \mathrm{C}$, the uncertainty of short-term stability at $40^{\circ} \mathrm{C}$ can be considered to be negligible.

3.3.2. Long-Term Stability Study. To satisfy the requirement of CRMs, the chloramphenicol in methanol, which was stored at $4^{\circ} \mathrm{C}$, was measured at 1st, 3rd, and 6th months. Longterm stability of chloramphenicol in methanol was examined by using the same analytical method and statistical analysis approach as described in short-term stability study. As shown in Table 6, no significance slope was found. This indicates that 


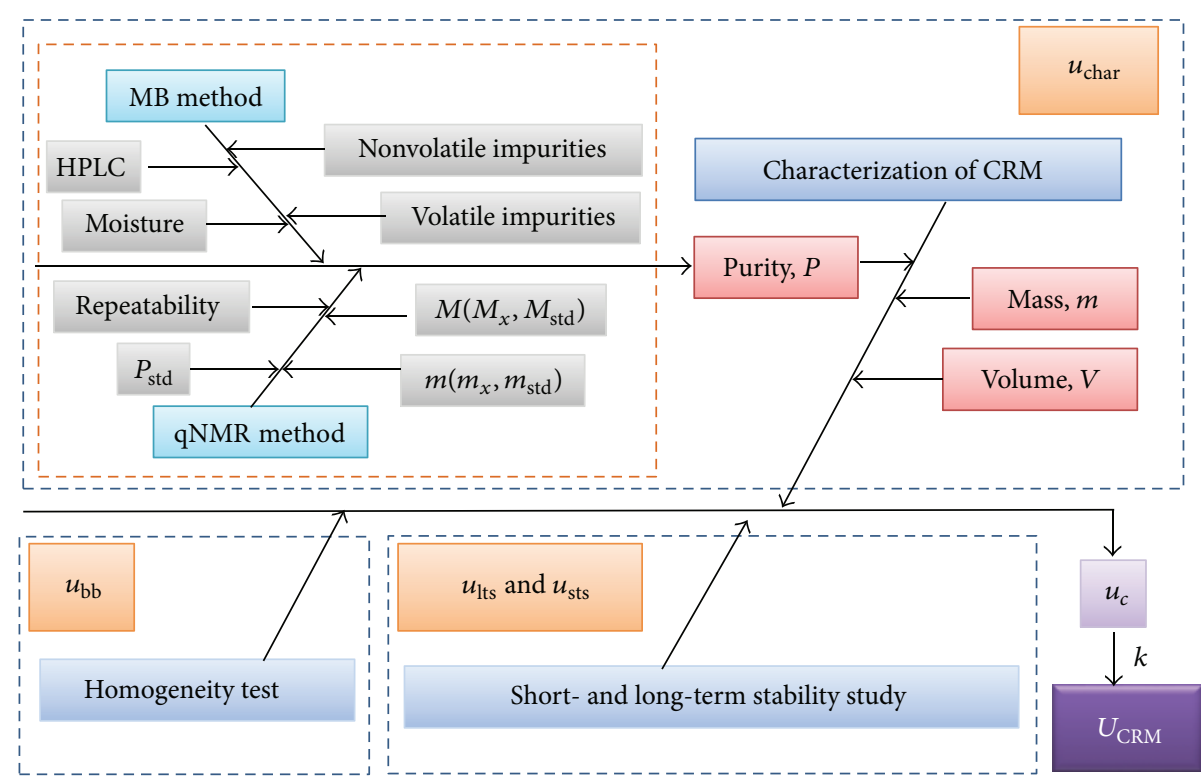

FIGURE 4: Uncertainty sources in the development process of chloramphenicol in methanol CRM.

TABLE 6: Results of long-term stability study.

\begin{tabular}{lcccc}
\hline \multirow{2}{*}{ Time/month } & \multicolumn{5}{c}{ Value/(mg/L) } \\
& 0 & 1 & 3 & 6 \\
\hline \multirow{3}{*}{100.4} & 100.4 & 101.4 & 100.2 \\
& 101.2 & 99.7 & 100.9 & 99.8 \\
& 101.1 & 99.6 & 101.0 & 100.5 \\
\hline 2 & 100.2 & 99.1 & 100.5 & 100.3 \\
& 101.6 & 101.4 & 100.3 & 99.9 \\
\hline Mean & 100.8 & 100.8 & 100.6 & 99.8 \\
\hline$b_{1}$ & 100.9 & 100.2 & 100.8 & 100.1 \\
$b_{0}$ & \multicolumn{5}{c}{-0.0845} \\
$s^{2}$ & \multicolumn{5}{c}{0.100 .7} \\
$s\left(b_{1}\right)$ & \multicolumn{5}{c}{0.0926} \\
$t_{0.95, n-2}$ & \multicolumn{5}{c}{4.3} \\
Conclusion & \multicolumn{5}{c}{$\mid<t_{0.95, n-2} \cdot s\left(b_{1}\right)$, stable } \\
\hline
\end{tabular}

chloramphenicol in methanol is stable for at least six months at $4^{\circ} \mathrm{C}$.

3.4. Evaluation of Uncertainties. In strict accordance with General and Statistical Principles for Characterization of Reference Materials JJF 1343-2012 [16], the sources of uncertainties of chloramphenicol in methanol CRM can be classified into three main parts (see Figure 4): uncertainty from homogeneity test $u_{\mathrm{bb}}$, uncertainties from long-term and short-term stability $u_{\mathrm{lts}}$ and $u_{\mathrm{sts}}$, and uncertainty from characterization $u_{\text {char }}$. Meantime, $u_{\text {char }}$ was comprised of the following items: balance precision, volume variation of volumetric flask, and purity of chloramphenicol material in which the uncertainty of purity was evaluated through combining uncertainties originating from purity determination process by two independent methods of mass balance and qNMR as shown in the orange dashed line rectangle in Figure 4.

For $\mathrm{MB}$ method, the standard uncertainty $u\left(P_{\mathrm{MB}}\right)$ can be calculated by the following equation:

$$
\begin{aligned}
& u\left(P_{\mathrm{MB}}\right) \\
& \quad=P_{\mathrm{MB}} \sqrt{\left(\frac{u\left(P_{0}\right)}{P_{0}}\right)^{2}+\frac{u^{2}\left(X_{\mathrm{w}}\right)+u^{2}\left(X_{\mathrm{n}}\right)+u^{2}\left(X_{\mathrm{v}}\right)}{\left(1-X_{\mathrm{w}}-X_{\mathrm{n}}-X_{\mathrm{v}}\right)^{2}}},
\end{aligned}
$$

in which $u\left(P_{0}\right)=u_{\text {HPLC }}$ can be evaluated by using the following equation:

$$
u_{\mathrm{HPLC}}=\sqrt{u_{1}^{2}+u_{2}^{2}}
$$

where $u_{1}$ is uncertainty originating from the standard deviation of HPLC-UV measurement; $u_{2}$ is uncertainty from difference of UV response factors of impurities, which can be estimated by the following equation:

$$
u_{2}=\frac{\sqrt{\sum_{i=1}^{n} u_{2-i}^{2}}}{\sqrt{3}}=\frac{\sqrt{\sum_{i=1}^{n}\left(B_{i \max \lambda}-B_{i \text { value } \lambda}\right)^{2}}}{\sqrt{3}},
$$

where $B_{i \max \lambda}$ is the maximum percentage of impurity $i$ at specific wavelength; $B_{i \text { value } \lambda}$ is the percentage of impurity $i$ at characterization wavelength; $u_{2-i}$ is uncertainty of impurity $i$. In this study, the wavelengths are selected at $210 \mathrm{~nm}, 235 \mathrm{~nm}$, $254 \mathrm{~nm}, 280 \mathrm{~nm}$, and $300 \mathrm{~nm}$.

Additionally, uncertainty of moisture measurement $u\left(X_{\mathrm{w}}\right)$ can be expanded by using the mean of measurements, respectively. In this case, the property value is more reliable and reasonable under the technical limitation. In addition, uncertainties of $u\left(X_{\mathrm{n}}\right)$ and $u\left(X_{\mathrm{v}}\right)$ can be ignored. 
TABLE 7: Results of uncertainty.

\begin{tabular}{|c|c|c|}
\hline Items & Assessment and calculation & Results \\
\hline \multirow{2}{*}{$u(m)$} & (1) variability of balance measurement (empirical estimation) & \multirow{2}{*}{$0.41 \%$} \\
\hline & (2) uncertainty of balance calibration (metrological verification certificate) & \\
\hline \multirow{3}{*}{$u(V)$} & (1) error of volumetric flask (metrological verification certificate) & \multirow{3}{*}{$0.35 \%$} \\
\hline & (2) random error (empirical estimation) & \\
\hline & (3) variation with temperature (calculation) & \\
\hline$u(P)_{\mathrm{rel}}$ & $u(P)_{\mathrm{rel}}=\frac{u(P)}{P}=\sqrt{\frac{u\left(P_{\mathrm{MB}}\right)^{2}}{P_{\mathrm{MB}}}+\frac{u\left(P_{\mathrm{NMR}}\right)^{2}}{P_{\mathrm{NMR}}}}$ & $0.28 \%$ \\
\hline$u_{\text {char,rel }}$ & $u_{\text {char }, \text { rel }}=\frac{u(c)}{c}=\sqrt{\left[\frac{u(P)}{P}\right]^{2}+\left[\frac{u(m)}{m}\right]^{2}+\left[\frac{u(V)}{V}\right]^{2}}$ & $0.61 \%$ \\
\hline$u_{\mathrm{bb}, \mathrm{rel}}$ & $u_{\mathrm{bb}, \mathrm{rel}}=\frac{\sqrt{\left(s_{1}^{2}-s_{2}^{2}\right) / n}}{\bar{X}_{\mathrm{bb}}}$ & $0.23 \%$ \\
\hline$u_{1 \mathrm{ts}} \& u_{\mathrm{sts}}$ & $u_{\mathrm{lts}, \mathrm{rel}}=s\left(b_{1}\right) \cdot t / \bar{X} \& u_{\mathrm{sts}, \mathrm{rel}}=s\left(b_{1}\right) \cdot t / \bar{X}$ & $0.56 \% \& 0.41 \%$ \\
\hline$u_{c, \text { rel }}$ & $u_{c, \text { rel }}=\sqrt{u_{\text {char,rel }}^{2}+u_{\mathrm{bb}, \text { rel }}^{2}+u_{\mathrm{lts}, \text { rel }}^{2}+u_{\mathrm{sts}, \text { rel }}^{2}}$ & $0.94 \%$ \\
\hline$U_{\mathrm{CRM}}$ & $U_{\mathrm{CRM}}=k \cdot u_{\mathrm{c}}=k \cdot c \cdot u_{c, \text { rel }} ;(k=2,95 \%$ confidence level $)$ & $2 \mathrm{mg} / \mathrm{L}$ \\
\hline
\end{tabular}

For qNMR, the standard uncertainty $u\left(P_{\mathrm{NMR}}\right)$ can be calculated by the following equation:

$$
u\left(P_{\mathrm{NMR}}\right)=P_{\mathrm{NMR}} \sqrt{\left(\frac{u\left(I_{x} / I_{\mathrm{std}}\right)}{I_{x} / I_{\mathrm{std}}}\right)^{2}+\left(\frac{u\left(M_{x}\right)}{M_{x}}\right)^{2}+\left(\frac{u\left(M_{\mathrm{std}}\right)}{M_{\mathrm{std}}}\right)^{2}+\left(\frac{u\left(m_{x}\right)}{m_{x}}\right)^{2}+\left(\frac{u\left(m_{\mathrm{std}}\right)}{m_{\mathrm{std}}}\right)^{2}+\left(\frac{u\left(P_{\mathrm{std}}\right)}{P_{\mathrm{std}}}\right)^{2}}
$$

where $u\left(I_{x} / I_{\text {std }}\right)$ was expressed by the relative standard deviation of measurement; $u\left(m_{\text {std }}\right)$ and $u\left(m_{x}\right)$ were from the balance weight; $u\left(P_{\text {std }}\right)$ was obtained from the certificate of ethyl paraben CRM (GBW(E)100064); $u\left(M_{x}\right)$ and $u\left(M_{\text {std }}\right)$ were calculated by the following equation:

$$
u(M)=\sqrt{\sum_{j=1}^{n}\left[N_{j} u_{j}\right]^{2}}
$$

where $N_{j}$ is atom number of $j$ element; $u_{j}$ is uncertainty of relative atomic mass of $j$ element which was cited from IUPAC table of atom weights of elements.

Therefore, uncertainty of purity measurement was evaluated as follows:

$$
u(P)=\sqrt{u\left(P_{\mathrm{MB}}\right)^{2}+u\left(P_{\mathrm{NMR}}\right)^{2}} .
$$

As we mentioned above, chloramphenicol in methanol was prepared by dissolving chloramphenicol into methanol. Thus, the concentration of CRM solution can be calculated by the following equation:

$$
c=\frac{1000 m P}{V}
$$

where $c$ is the concentration of chloramphenicol in methanol $(\mathrm{mg} / \mathrm{L}) ; m$ is amount of chloramphenicol $(\mathrm{mg}) ; P$ is the purity of chloramphenicol; $V$ is volume of methanol $(\mathrm{mL})$.
The relative uncertainty of characterization can be expressed by the following equation:

$$
\begin{aligned}
u_{\text {char,rel }} & =\frac{u(c)}{c} \\
& =\sqrt{\left[\frac{u(P)}{P}\right]^{2}+\left[\frac{u(m)}{m}\right]^{2}+\left[\frac{u(V)}{V}\right]^{2}} .
\end{aligned}
$$

According to above analysis of uncertainty, the evaluated uncertainty results are summarized in Table 7.

3.5. Value Verification. In order to check up the reliability and accuracy of the assigned value of chloramphenicol in methanol CRM in this development project, an existing certified reference material of chloramphenicol (GBW(E)060907) was selected as calibrator to measure the property value of chloramphenicol in methanol CRM. A calibration solution of chloramphenicol with a concentration of $100.9 \mathrm{mg} / \mathrm{L}$, which is approximate to the assigned value of $(100 \pm 2) \mathrm{mg} / \mathrm{L}$, was precisely prepared by verified balance and volumetric flask. The developed CRM of chloramphenicol in methanol as an unknown sample was measured in triplicate. As shown in Table 8 , the mean of measured value is $99.7 \mathrm{mg} / \mathrm{L}$ with a standard deviation of $0.2 \mathrm{mg} / \mathrm{L}$. It was found that the measured value was covered by the assigned value accurately. Therefore, it can be approved that the assigned value of chloramphenicol in methanol was reliable and accurate. 
TABLE 8: Verification results of chloramphenicol in methanol CRM (unit: $\mathrm{mg} / \mathrm{L}$ ).

\begin{tabular}{ccccccc}
\hline \multirow{2}{*}{ Item } & \multicolumn{5}{c}{ Measured value } & \multirow{2}{*}{ Assigned value } \\
& 1 & 2 & 3 & Mean & SD & \\
\hline CRM & 99.5 & 99.9 & 99.7 & 99.7 & 0.2 & $100 \pm 2$ \\
\hline
\end{tabular}

\section{Conclusion}

A user-friendly and time-saving CRM of chloramphenicol in methanol (GBW(E)082557) was successfully developed and was approved by General Administration of Quality Supervision, Inspection, and Quarantine of China. In the study, the qNMR and mass balance methods were employed in the precise measurement of chloramphenicol purity. The homogeneity, stability, and uncertainty of this CRM were sufficiently studied according to the technical specification. In addition, the selection of proton peak in qNMR spectrum for purity measurement was comparatively studied.

\section{Competing Interests}

The authors declare that there are no competing interests regarding the publication of this paper.

\section{Acknowledgments}

The authors acknowledge the financial support from the Ministry of Agriculture of China (Special Fund for AgroScientific Research GJFP201501503) and the Innovation Workshop Project of Chinese Academy of Agricultural Sciences.

\section{References}

[1] T. Yamazaki, T. Watanabe, S. Nakamura, and K. Kato, "Purity determination of acetaldehyde in an acetaldehyde certified reference material," Analytical Sciences, vol. 31, no. 6, pp. 463468, 2015.

[2] H. Gong, T. Huang, Y. Yang, and H. Wang, "Purity determination and uncertainty evaluation of folic acid by mass balance method," Talanta, vol. 101, pp. 96-103, 2012.

[3] K. Ishikawa, N. Hanari, Y. Shimizu et al., "Mass balance method for purity assay of phthalic acid esters: development of primary reference materials as traceability sources in the Japan Calibration Service System," Accreditation and Quality Assurance, vol. 16, no. 6, pp. 311-322, 2011.

[4] S.-H. Kim, J. Lee, S. Ahn, Y.-S. Song, D.-K. Kim, and B. Kim, "Purity assessment of organic reference materials with a mass balance method: a case study of endosulfan-II," Bulletin of the Korean Chemical Society, vol. 34, no. 2, pp. 531-538, 2013.

[5] K. Ma, H. Wang, M. Zhao, and J. Xing, "Purity determination and uncertainty evaluation of theophylline by mass balance method, high performance liquid chromatography and differential scanning calorimetry," Analytica Chimica Acta, vol. 650, no. 2, pp. 227-233, 2009.

[6] Y. Shimizu, Y. Ohte, and K. Kato, "Development of a certified reference material for calibration of DSC and DTA below room temperature: NMIJ CRM 5401-a, Cyclohexane for Thermal Analysis," Thermochimica Acta, vol. 568, pp. 61-66, 2013.

[7] S. Mathkar, S. Kumar, A. Bystol et al., "The use of differential scanning calorimetry for the purity verification of pharmaceutical reference standards," Journal of Pharmaceutical and Biomedical Analysis, vol. 49, no. 3, pp. 627-631, 2009.

[8] A. Le Gresley, F. Fardus, and J. Warren, "Bias and uncertainty in non-ideal qNMR analysis," Critical Reviews in Analytical Chemistry, vol. 45, no. 4, pp. 300-310, 2015.

[9] T. Huang, W. Zhang, X. Dai et al., "Precise measurement for the purity of amino acid and peptide using quantitative nuclear magnetic resonance," Talanta, vol. 125, pp. 94-101, 2014.

[10] R. Nogueira, B. C. Garrido, R. M. Borges, G. E. B. Silva, S. M. Queiroz, and V. S. Cunha, "Development of a new sodium diclofenac certified reference material using the mass balance approach and ${ }^{1} \mathrm{H}$ qNMR to determine the certified property value," European Journal of Pharmaceutical Sciences, vol. 48, no. 3, pp. 502-513, 2013.

[11] G. F. Pauli, S. N. Chen, C. Simmler et al., "Importance of purity evaluation and the potential of quantitative ${ }^{1} \mathrm{H}$ NMR as a purity assay," Journal of Medicinal Chemistry, vol. 57, no. 22, pp. 9220 9231, 2014.

[12] C. Quan, "Establishment of the purity values of carbohydrate certified reference materials using quantitative nuclear magnetic resonance and mass balance approach," Food Chemistry, vol. 153, pp. 378-386, 2014.

[13] "General requirements for the competence of reference material producers," ISO Guide 34, International Organization for Standardization, Geneva, Switzerland, 2009.

[14] ISO Guide 35, Reference Material-General and Statistical Principles for Certification, International Organization for Standardization, Geneva, Switzerland, 2006.

[15] "Chinese technical norm of primary reference material," JJF 1006-1994, General Administration of Quality Supervision, Inspection, and Quarantine, Beijing, China, 1994.

[16] General Administration of Quality Supervision, Inspection, and Quarantine, "General and statistical principles for characterization of reference materials," JJF 1343-2012, General Administration of Quality Supervision, Inspection, and Quarantine, Beijing, China, 2012. 

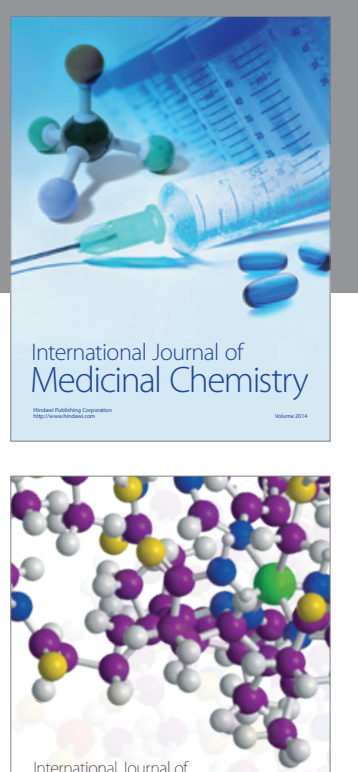

Carbohydrate Chemistry

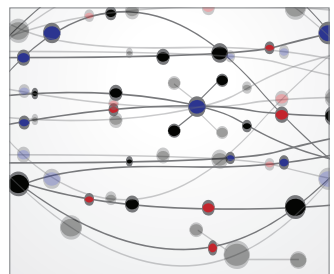

The Scientific World Journal
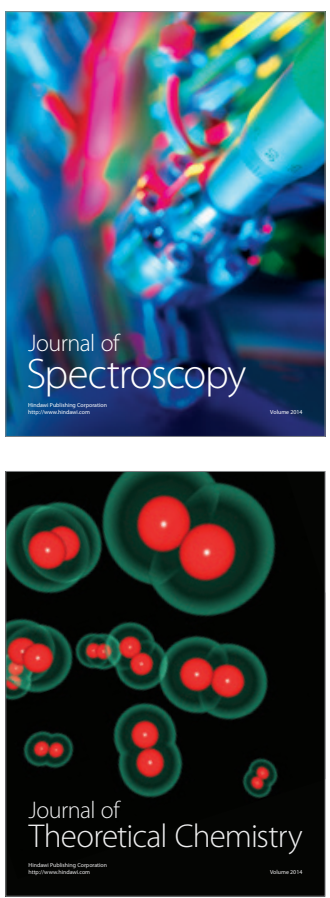
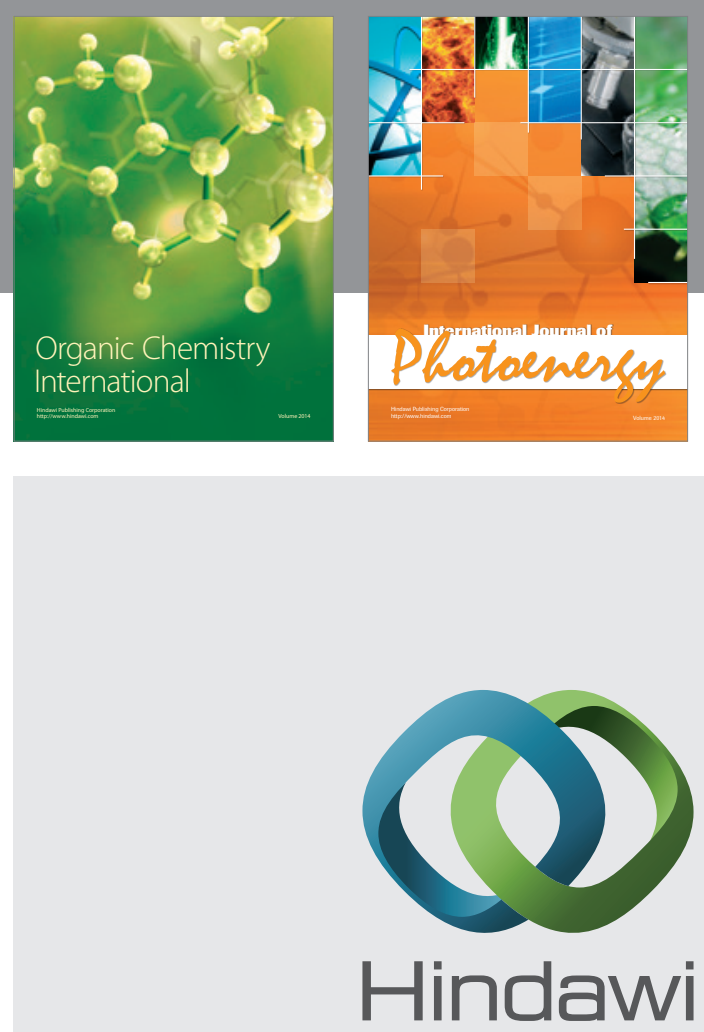

Submit your manuscripts at

http://www.hindawi.com

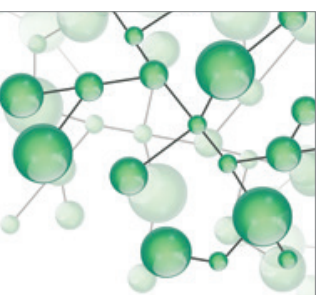

International Journal of

Inorganic Chemistry

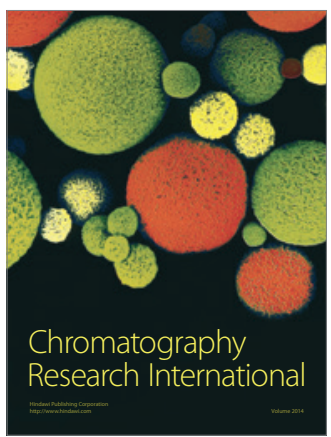

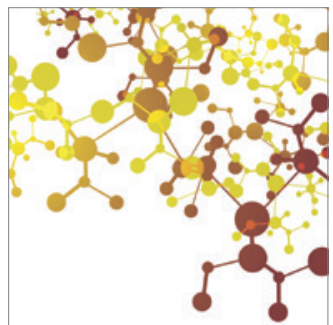

Applied Chemistry
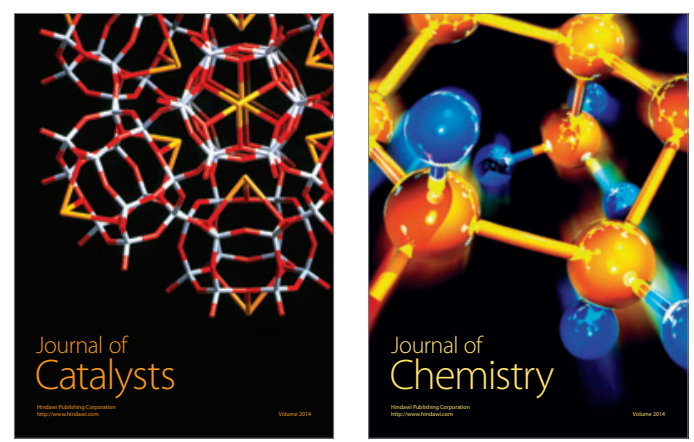
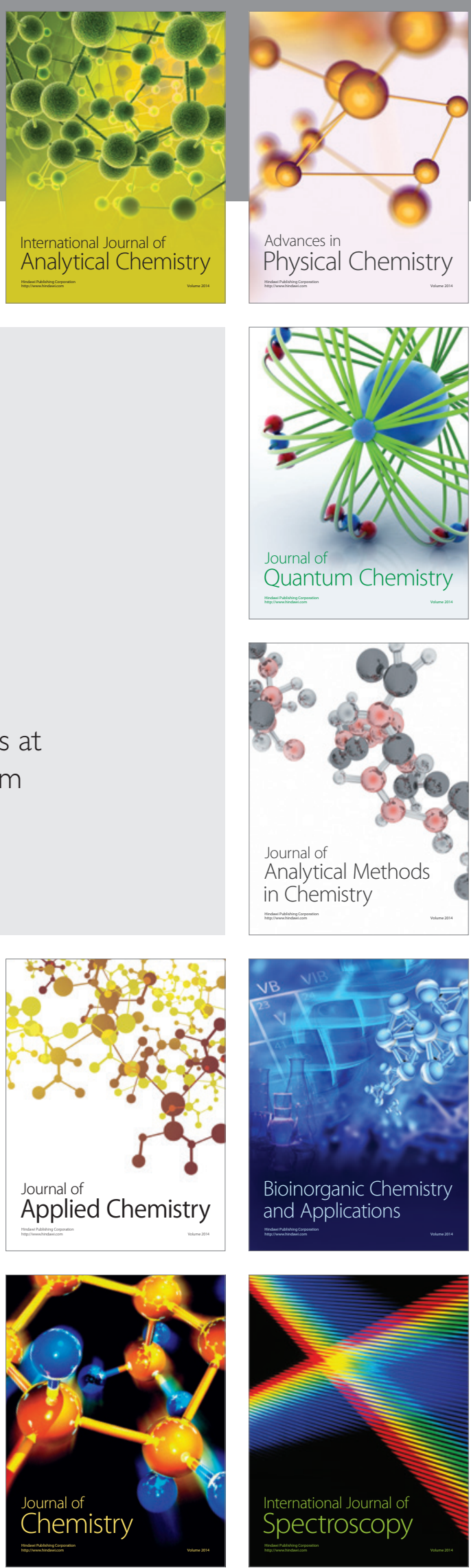